\title{
COLLOIDS IN SEPTIC TANK EFFLUENT AND THEIR INFLUENCE ON FILTER PERMEABILITY
}

\author{
Marcin Spychała', Jakub Nieć' , Natalia Walczak' ${ }^{1}$, Anna Marciniak ${ }^{2}$
}

1 Faculty of Land Reclamation and Environmental Engineering Department of Hydraulic and Sanitary Engineering, University of Life Sciences in Poznan, Wojska Polskiego St. 28, 60-637 Poznań, Poland, e-mai: marsp@ au.poznan.pl; jniec@up.poznan.pl; natwal@up.poznan.pl

2 Firma Instalacyjno-Budowlana Jerzy Czwordon, Powstania Listopadowego St. 20, 63-400 Ostrów Wielkopolski, Poland, e-mail: anna.marciniak1989@gmail.com

Received: 2015.06.02

Accepted: 2015.08.31

Published: 2015.10.01

\begin{abstract}
The aim of the study was to evaluate the content of colloids in septic tank effluent and their impact on textile filter permeability. Measurements were performed on septic tank effluent without suspended solids but containing colloids and without colloids - including only dissolved substances (filtered by micro-filters and centrifuged). The study was conducted on unclogged and clogged textile filter coupons. During the study the following measurements were conducted: turbidity, chemical oxygen demand and hydraulic conductivity of textile filter coupons. The colloid size range was assumed to be less than 1.2 microns according to the literature. Despite the relatively low content in the septic tank effluent the colloids played an important role in the clogging process. Both the filtering media, filled with low (unclogged) and high content of biomass (clogged) were sensitive to the clogging process acceleration due to the possibility of small diameter pore closure and oxygen access termination. Moreover, small size pores were probably sensitive to closing or bridging by small size colloidal particles.
\end{abstract}

Keywords: colloids, clogging, hydraulic conductivity, non-woven textile, turbidity.

\section{INTRODUCTION}

Colloids are particles of diameter measuring $1 \mathrm{~nm}-1$ micrometer and can be observed to be very common in nature. These include contaminants in the water, milk (protein and fat droplets in water), butter (colloidal fraction of water in a fat), protein solutions, jellies, mist (water drops or suspension ice in the air) and others. The concept of a colloidal substance refers to emulsions, suspensions present in the water, fibers, polymers and fumes [Górski 1953].

Whereas the solutes in wastewater have mainly an indirect effect on the permeability of the filter acting on biomass growth, solid and colloidal particles are a direct mechanical factor limiting permeability. However, the septic tank effluent (STE) content and the mechanisms of particle retention on or inside the filter are poorly recognized.
Colloids are commonly found, as well as liquids, solids and gases. They can be characterized by a specific range of fragmentation of the components contained therein. Colloidal particles are unable to pass through the biological membrane (cell membrane). However, it should be noted that they belong to a heterogeneous mixture in the form of suspension [Drapała 1996]. The diversity of colloids makes it difficult to formulate a clear definition of colloidal particles.

It has been shown that substances may take the form of colloids, suspensions, or a real solution, depending on the dispersion media and the fragmentation of the mixture [Drapała 1996].

The scholarly literature indicates a common assumption that colloidal particles have a size of less than about 1 micron, while some authors reported a maximum dimension of $0.45 \mu \mathrm{m}$ and even an upper limit of 10 microns [Perez-Paricio 
and Carrera 1999]. In most cases they are characterized by a very high surface area, resulting in the suspension of colloidal particles impacted by surface physico-chemical forces; in particular such as the attraction of London-van der Waals and electrostatic attractive forces. The distance of impact is very small, and the effects are significant when the particles cross the energy barrier [Smith 2007].

Brownian diffusion also plays an important role in the behavior of these particles in porous media. In comparison with real solutions, colloidal suspensions consist of much bigger particles, thereby extending the time of diffusion and contributing to a low osmotic pressure of the solution in question. A careful analysis of the optical properties can assess how it is affected by media dispersion. This phenomenon is known as the dispersion Tyndall effect where the light beam behaves quite differently in the case of colloidal suspensions than in real solutions that exhibit optical homogeneity. In the former case it is dissipated by the dispersion medium. One example would be the sunlight passing through a narrow gap where the dust floating in space scatters the beam of sunlight showing its path. It should aslo be noted that the dispersion of light takes place in various directions [Bijok and Bijok 1974].

In raw wastewater colloids may reach up to $21-22 \%$ of chemical oxygen demand (COD) and most are (up to $19-20 \%$ of COD) readily biodegradable [Makowska and Spychała 2014]. The high range of biodegradable fraction of colloids can result in their concentration changeability.

Kubiak [2014] reported colloids as a COD content (identified as the difference between domestic wastewater filtered by $1.0 \mu \mathrm{m}$ and $0.22 \mu \mathrm{m}$ microfilters) as being between $0.6 \%$ and $8.2 \%$, on average: $5.5 \pm 1.75 \%(n=4)$. This value was calculated by excluding values below zero (three of seven).

The particles of size 1-30 microns are considered as intermediate particles [Perez-Paricio and Carrera 1999] and are therefore influenced by both the particles and volume of surface forces. Some authors assume that surface forces can be omitted in the case of particles larger than $10 \mathrm{mi}$ crons [Sanford and Gates 1956]. Other researchers draw attention to the role of van der Waals forces [Mintz 1964, Mackrle 1960] and even that electro-kinetic forces should be taken into account [Stanley 1955, O'Melia and Crapps 1964, Ives 1965]. In the general context, the sedimentation of these particles is questioned or considered by some authors [Camp 1937, Fair 1951, Ives 1961] and ignored by others [Stein 1940, O'Melia and Crapps 1964, Smith 1967].

For large particles, it is believed that the size (greater than 30 microns) is subject only to the forces of volume in respect to process sedimentation, straining, and hydrodynamic conditions [Perez-Paricio and Carrera 1999].

Comprehensive information on a particular sample (e.g. colloids) cannot be based solely on one method. The following are used for the determination of colloids. Nephelometry and turbidimetry, which allows the determination of the function of particle size distribution in respect to the dispersed phase of substances. In this context light beam scattering plays a decisive role.

Ultra-microscopy, which is applicable only to a solution showing the Tyndall effect and is relevant for liophobic substances, where the difference between the refractive factor of the medium and the refractive factor of the system (constituted by colloidal particles) is significant.

Electron microscopy, involving the use of an electron beam instead of visible light, where the electron beam is characterized by a small wavelength. For this purpose, lenses have been replaced by an electromagnetic field, which allows for the collapsing of the electron pathway. The two most popular types of electron microscope are scanning and transmission.

Ultracentrifuge, is centrifuge performing for several or tens of thousands rotations. Due to the different particle size distribution in the STE and due to the presence of relatively small particles (several micrometers), the possibility of the formation of the smallest particle aggregates (less than 10 microns) by the action of surface forces (van der Waals) or electro-kinetic [Malinowskaja et al. 1986] should be taken into consideration. An aggregate may behave as a single particle or as a floc, which is unstable and undergoes deformation under the influence of external forces such as a pressure gradient. A disadvantage of septic tank (ST) outflow is its high variability in terms of both quantity and quality.

The composition of the STE has an impact on the quality and durability of filters (e.g. non-woven) correspondingly both to dissolved and suspended substances. Wherein the former (as easily absorbed) have an impact on the development of live biomass, while the latter - act on permeability reduction - as the result of accumulation in the filter pores (clogging) or on the surface (forma- 
tion of a filter cake). Previous studies on the STE showed the high differentiation of total suspended solids (TSS) concentration [Spychała et al. 2013]. Until now some guidelines for acceptable maximum TSS concentration for sand filters supplied with STE have been proposed. The effect of particle size and shape distribution on the filter (sand or non-woven textile filters) is practically unrecognized in the pore blocking (clogging) process. Particle size distribution in STE and their qualitative relation to the raw wastewater inflowing into the ST is also little known. In opposition to most sand filters non-woven textile ones have a relatively small available pore size, therefore the impact of particle size on filter performance (hydraulic capacity) is all the more important.

The particles in the wastewater (including STE) can be distinguished into several size fractions [Dulekgurgen et al. 2006]: dissolved $(<0.001 \mu \mathrm{m})$, colloidal $(0.001-1 \mu \mathrm{m})$, "supra" colloidal $(1-100 \mu \mathrm{m})$ and settling $(>100 \mu \mathrm{m})$.

To date, several studies were carried out on the determination of particle size distribution in municipal, industrial and agricultural wastewater but not in the STE [Sophonsiri and Morgenroth, 2004]. Authors investigated the relationship between chemical composition and particle size distribution. In this context one should note that total suspended solids are composed mainly of organic matter and have a density of about $1 \mathrm{~g} / \mathrm{cm}^{3}$ or less, which makes it relatively difficult to remove by sedimentation and easier to remove by filtration.

The suspended solids concentration determined by the filtration on a cellulose filter can provide important information about the minimum particle size as long as the pore size of the filter is known and reported. However, this is not common situation. Additionally the complication is that the guidelines in various countries differ in this matter. Despite the relatively high proportion of the organic fraction in suspension in general, some authors suggest that this is a matter of very low susceptibility to degradation [Siegrist 1987], where it was found that about half of the total suspended solids in STE is low-biodegradable or inert.

The STE contains many kinds of particles, originating from various materials such as toilet paper, tampons, paper napkins, paper towels, cotton or wool fibers, cigarette filters, and many others [Friedler et al. 1996, Seabloom et al. 2005].

One of the more frequently occurring particles in the STE are fibers or particles of a fibrous shape [Spychała et al. 2013], including fibers derived from toilet paper.

Some published studies [Friedler et al. 1996] indicate a relatively high proportion by weight of waste toilet paper produced by one person per day on average - more than 10\% (13 paper leaves, $0.55 \mathrm{~g}$ of weight each giving about $7.2 \mathrm{~g}$ dry weight per person per day). The interpretation of these results is difficult due to the probable large variation of the amount of paper wasted by one person, which depends on various factors, such as gender [Friedler et al. 1996] or age [van der Wijst and Groot-Marcus 1999], and perhaps also other individual characteristics.

Jönsson et al. [2005] showed that $48 \%$ of the weight of toilet paper consists of carbon, and the fibers are composed mainly of cellulose and hemicellulose. These authors also determined the rate of medium and slowly degradable carbohydrates in toilet paper ( $83 \%$ and $17 \%$, respectively).

Becker [1994] and Becker and Pearson [2002] estimated the average daily weight of fiber in the Swedish diet, of $15.0 \mathrm{~g} / \mathrm{PE}$ and $17.0 \mathrm{~g} / \mathrm{PE}$, respectively. To date the published research results on particle size distribution of suspended solids in the STE have been few and far between. One of the more interesting results was presented by Pallares et al. [2011]. These studies have shown a relatively wide range of the length of fibrous particles originating from toilet paper: $180-540 \mu \mathrm{m}$.

The size distribution of particles occurring or those likely to be present in the STE is characterized by a high variability in both the average and ranges of values. Sizes are in the range from a few to several hundred. In practical terms the probability of non-woven textile pore blockage (typical pore size of medium thickness non-woven textiles is about $90-120 \mu \mathrm{m}$ ) at the surface of non-woven textile in the form of a filter cake can occur in the case of toilet paper fibers and large fractions of potato starch. Knowledge related to particle size and shape distribution in the STE appears to be necessary for the design and modelling of filters, including non-woven textile ones [Pallares et al. 2011], as well as for the identification of processes, including biological filters performance [Dulekgurgen et al. 2006, Levine et al. 1991, Sophonsiri and Morgenroth 2004]. Such knowledge has been seen to be also useful for biochemical process description, e.g. for the relationship between particle size distribution and the COD of the organic substrate [Dulekgurgen et al. 2006, Ekama et al. 1986, Henze et al. 2000]. 
Another important problem is the content of non-fibrous particles originating from toilet paper in the STE [Spychała et al. 2013].

The most of particles kinds referred above can change into the colloids under specific conditions, e.g. as the result of the composition by relatively high bacteria number.

The aim of the study was to verify the hypothesis that colloids can prove to be a significant factor in filter permeability.

\section{METHODS}

The research was conducted on wastewater collected from a single chamber septic tank of $3.0 \mathrm{~m}^{3}$ volume (length: $1.85 \mathrm{~m}$, width: $1.34 \mathrm{~m}$ ) equipped with an outflow filter. The STE originated from a four-people household (two adults and two children). The STE was initially filtered by a filter of $10 \mu \mathrm{m}$ pores. The turbidity and COD was measured in each sample.

\section{Hydraulic conductivity determination}

Aiming to avoid damage or disturbance of the clogging layer, the STE level at the start of measurement was sustained $10 \mathrm{~cm}$ above the filter layer surface. Six transparent experimental pipes were used (three unclogged textile filters and three clogged textile filters). After the stable water surface level reached the measurements of water surface level fall-time were performed for STE with and without colloids. All the measurements were done in several from 5 to 8 replications.

The flow rate and hydraulic conductivity calculation was based on the determination of falling water surface level during filtration through the clogged and unclogged non-woven textile filter (four layers of TS 20 textile type). The hydraulic conductivity was determined and calculated according to equation no. (1) presented by Lambe [1987], using the method described by Li et al. [2005] and confirmed by Nieć and Spychała [2014]:

$$
K_{u s}=2.3 \frac{l}{t} \log \left(\frac{H_{0}}{H_{t}}\right)
$$

where: $K_{u s}$ - hydraulic conductivity; estimated at unsteady state flow, $\mathrm{m} / \mathrm{d}$;

$l$ - thickness of filtration layer, $\mathrm{m}$;

$t$ - fall-time of water surface level from $H_{o}$ to $H_{t}$, d;

$H_{o}^{o}$ - height of water surface level at the start of measurement, m;

$H_{t}$ - height of water surface level at the end of measurement, $\mathrm{m}$.

After filtration by filter of $10 \mu \mathrm{m}$ pores (removal of TSS) the STE was filtered by microporous filter of pores $1.2 \mu \mathrm{m}$ and then centrifuged during 30 minutes at 6000 RPM. Measurements were performed in two series: unclogged-textile filtration series and clogged-textile filtration series. The basic information related to sample data and methods was collected in Table 1.

The hydraulic load of textile filter coupons with filter STE without colloids (filtered by $1.2 \mu \mathrm{m}$ micro filters and centrifuged) and those with colloids (filtered by $1.2 \mu \mathrm{m}$ micro filters) was $30 \mathrm{~cm}^{3}$ and $26 \mathrm{~cm}^{3}$, respectively.

The surface area of filter layer sample during unclogged non-woven textile filtration experiment was $0.5 \mathrm{~cm}^{2}$. The filters were practically free of TSS. Only about $180 \mathrm{~cm}^{3}$ of STE was filtered by filters before the unclogged non-woven textile experiment for the purposes of wetting the filtering layer.

The research on clogged filter was conducted on a transparent pipes with an $0.8 \mathrm{~cm}$ diameter and $40 \mathrm{~cm}$ in height. The surface area of clogged filter coupons was $0.83 \pm 0.5 \mathrm{~cm}^{2}$. The clogged non-woven textile was fastened at the base edge of the pipes. Accumulated mass (measured as TS) on the surface and inside the filter layer sample $24.0 \pm 2.87 \mathrm{~cm}^{2}$ on average indicated an advanced clogging process.

Table 1. The basic information related to sample data and methods

\begin{tabular}{|l|l|c|c|}
\hline \multirow{2}{*}{ Series } & \multicolumn{2}{|c|}{ Septic tank effluent characteristics } \\
\cline { 2 - 4 } & \multicolumn{1}{|c|}{ Size fraction } & $\mathrm{COD}, \mathrm{gO}_{2} / \mathrm{m}^{3}$ & Turbidity, FAU \\
\hline \multirow{2}{*}{ Unclogged-textile } & Filtered by $10 \mu \mathrm{m}$ filter & $838.75 \pm 72.45$ & 100 \\
& filtered by micro filter $(1.2 \mu \mathrm{m})$ & $723.00 \pm 37.24$ & $>100$ \\
\hline \multirow{2}{*}{ Clogged-textile } & Filtered by $10 \mu \mathrm{m}$ filter & $752.50 \pm 22.98$ & $24.33 \pm 4.78$ \\
\hline & Filtered by micro filter $(1.2 \mu \mathrm{m})$ & $606.25 \pm 8.44$ & \\
\hline
\end{tabular}




\section{RESULT AND DISCUSSION}

\section{Colloids content in septic tank effluent}

The colloids were identified by COD and turbidity difference between STE filtered by a 1.2 $\mu \mathrm{m}$ micro filter (without suspended solids but with colloids) and STE centrifuged (without suspended solids and without colloids).

A significant difference in COD between STE with colloids and without colloids was observed during clogged-textile filtration experiment: $606.25 \pm 8.44 \mathrm{gO}_{2} / \mathrm{m}^{3}$ and $562.50 \pm 9.56 \mathrm{gO}_{2} / \mathrm{m}^{3}$ respectively (Figure 1). Unfortunately no statistically significant difference was observed in COD between STE with colloids and STE without colloids during unclogged textile filtration experiment. An evident difference was observed in colloids content identified by turbidity between STE with colloids and STE without colloids during unclogged and clogged textile filtration experi-

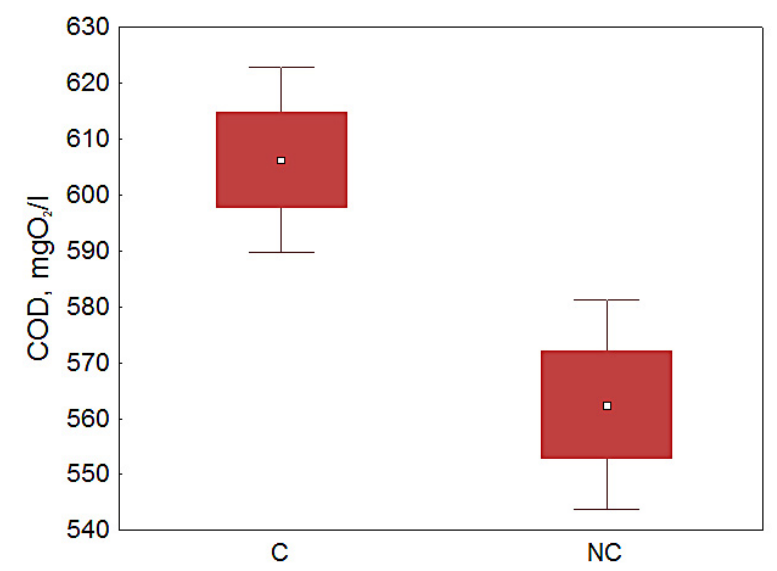

Figure 1. COD in septic tank effluent with colloids (C) and without colloids (NC) clogged non-woven textile filter experiment

a)

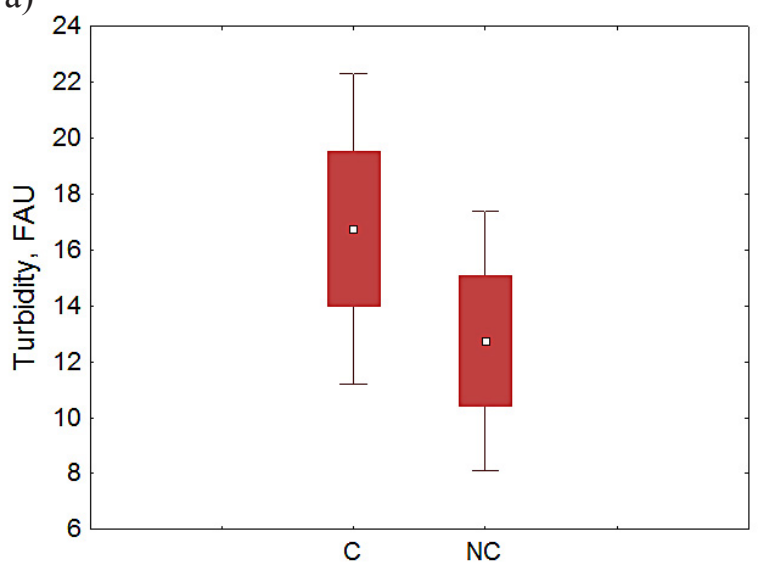

ment: $24 \%$ and $14 \%$, respectively. The results are presented in Figure 2.

Statistical analysis ( $t$ - student's test) at the significance level $\alpha=0.05$, confirmed a significant difference in turbidity between STE with colloids (C) and STE without colloids (NC) in both, unclogged and clogged textile filtration experiment.

The observed content of colloids in STE, both during unclogged and clogged textile filtration experiment were relatively low and unstable. This corresponds to results obtained by Kubiak [2014] and was probably related to raw wastewater content (wastewater inflowing into the ST) and biochemical processes occurring inside the ST. Importantly, these processes have not been fully recognized yet.

\section{Colloids impact on unclogged and clogged textile filter hydraulic conductivity}

The hydraulic conductivity of filters number 2 and number 3 (Figure 3) confirmed (at the level $\alpha=0.05$ ) the significant impact of colloids on unclogged and clogged textile filter hydraulic conductivity (e.g. filter number 2 without and with colloids: $0.186 \pm 0.003$ and $0.132 \pm 0.002 \mathrm{~m} / \mathrm{d}$, respectively, filter number 3 without and with colloids: $0.052 \pm 0.003$ and $0.036 \pm 0.001 \mathrm{~m} / \mathrm{d}$, respectively). Unfortunately the results of hydraulic conductivity of filter number one did not show the significant impact of colloids (at the level $\alpha=0.05$ ). This could be related to the local variability of textile porosity. This problem was earlier reported by Spychała and Łucyk [2015, in press].

Despite the relatively low content in the STE, colloids can play an important role in the

b)

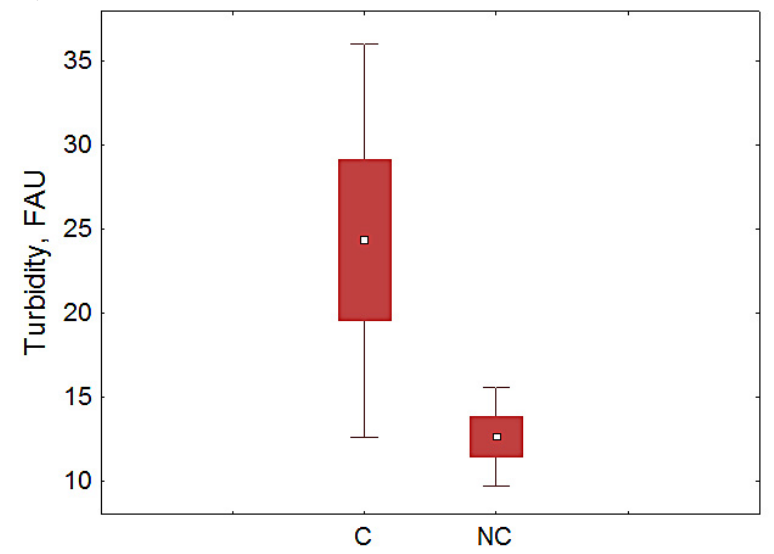

Figure 2. Turbidity in septic tank effluent with colloids (C) and without colloids (NC) during unclogged nonwoven textile filter (left) clogged non-woven textile filter (right) experiment 
a)

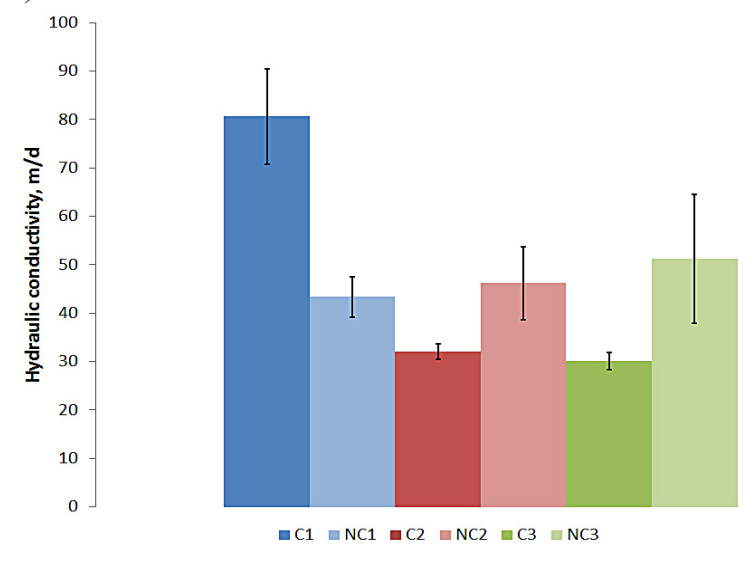

b)

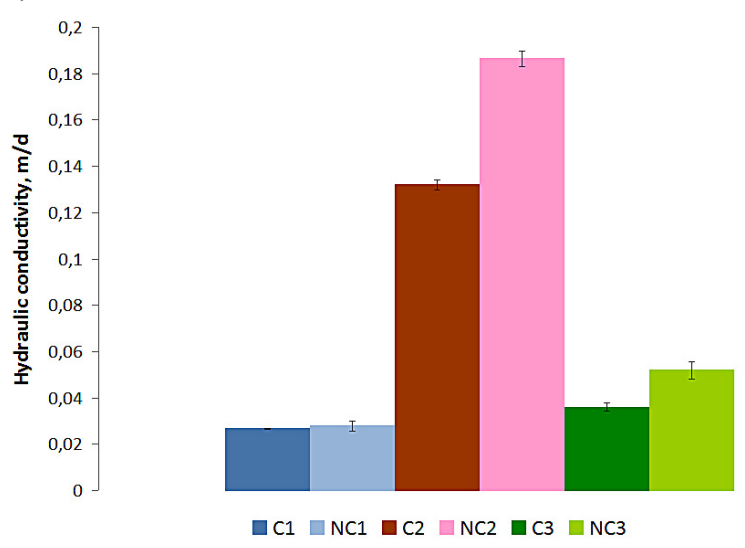

Figure 3. Hydraulic conductivity of filters supplied with STE containing colloids (C), and not containing (NC) colloids; left: unclogged non-woven textile filtration experiment, right: clogged non-woven textile filtration experiment

clogging process. Filtering media filled with a high content of biomass (partly clogged) is sensitive to clogging process acceleration due to the possibility of small diameter pore blockage and oxygen access termination. Moreover, small size pores are sensitive to blocking or bridging by small size particles. The impact of STE with and without colloids on the permeability of unclogged and clogged non-woven textile was observed.

\section{CONCLUSIONS}

The following conclusions can be drawn based on this research:

- the observed values and content of colloids in STE, both during unclogged and clogged nonwoven textile filtration experiment was relatively low and unstable

- the identification of colloids by turbidity (fractionation using filtration and centrifugation) was more appropriate and effective than identification by COD due to some instability of organic compounds and lack of detection of inorganic colloid compounds

- despite the relatively low content in the STE, colloids can play an important role in nonwoven textile filter clogging process due to the possibility of small diameter pores closing by (colloidal particles) and oxygen access stopping

- the identification and description of colloid accumulation process during STE filtration (e.g. by non-woven textile filters for wastewater treatment) needs further studies.

\section{Acknowledgements}

This work was supported by the National Science Centre under Grant No. N N523751540.

\section{REFERENCES}

1. Becker, W. 1994. Dietary habits and intake of nutrition in Sweden 1989 (In Swedish). The National Food Administration. Uppsala, Sweden.

2. Bijok B., Bijok F. 1974. Organic chemistry for food industry secondary schools. WSiP, Warsaw.

3. Górski A. 1953. Colloids science outline. PWRiL, Warsaw.

4. Drapała T. 1996. Chemistry fundamentals. WSP, Warsaw.

5. Dulekgurgen E., Doğruel S., Karahan Ö., Orhon D. 2006. Size distribution of wastewater COD fractions as an index for biodegradability. Water Res. 40 (2), 273-282.

6. Ekama G.A., Dold P.L., Marais G.V.R. 1986. Procedures for determination of influent COD fractions and the maximum specific growth rate of heterotrophs in activated sludge system. Water Sci. Technol. 18, 91-114.

7. Fair G.M 1951. The hydraulics of rapid sand filters. J. Inst. of Water Eng. 5, 171-213.

8. Friedler E., Brown D.M., Butler D. 1996. A study of WC derived sewer solids. Water Sci Technol. 33(9), 17-24.

9. Kubiak M. 2014. Comparison of fractionation methods of domestic wastewater contaminants. Master of science thesis. University of Life Sciences in Poznan.

10. Lambe T.W. 1978. Soil Mechanics. Arkady, Warsaw. 
11. Li W., Kiser C., Richard Q. 2005. Development of a filter cake permeability test methodology. American Filtration and Separations Society. International Topical Conferences and Exposition, September 19-22, Ann Arbor, Michigan, (MI), p. 1-8.

12. Makowska M., Spychała M. 2014. Organic compounds fractionation for domestic wastewater treatment modeling. Pol. J. Environ. Stud. 23(1), 131-137.

13. Malinowskaja T.A. 1986. Solids separation in chemical industry (In Polish). Science Technical Press. Warsaw.

14. Nieć, J.; Spychała, M. 2014. Hydraulic Conductivity Estimation Test Impact on Long-Term Acceptance Rate and Soil Absorption System Design. Water, 6, 2808-2820.

15. O’Melia C.R., Crapps D.K. 1964. Some Chemical Aspects of Rapid Sand Filtration. Annual Conference, Toronto, Ont. Jun. 4.

16. Pallarès A., François P., Pons M.N., Schmitt P. 2011. Suspended particles in wastewater: their optical, sedimentation and acoustical characterization and modeling. Water Sci. Technol. 63(2), 240-247.

17. Perez-Paricio A., Carrera J. 2000. Validity and sensitivity analysis of a new comprehensive clogging model. Calibration and Reliability in Groundwater Modelling: Proceedings of the ModelCARE 99 Conference, pp. 47-53, Int. Assoc. of Hydrol. Sci., Gentbrugge, Belgium.
18. Sanford L.H., Gates C.D. 1956. Effects of Synthetic Detergents on Rapid Sand Filter Performance. J. AWWA, 45-54.

19. Seabloom R.W., Bounds T.R., Loudon T.L. 2005. Septic tanks. In: Gross M.A., Deal N.E. (Eds.) University curriculum development for decentralized wastewater management. National Decentralized Water Resources Capacity Development Project. Fayetteville (AR), University of Arkansas, 38-39.

20. Siegrist R.L. 1987. Soil clogging during subsurface wastewater infiltration as affected by effluent composition and loading rate. J. Environ. Qual. 16(2), 181-187.

21. Sophonsiri C., Morgenroth E. 2004. Chemical composition associated with different particle size fractions in municipal, industrial, and agricultural wastewaters. Chemosphere, 55(5), 691-703.

22. Spychała M., Nieć J. 2013. Impact of septic tank sludge on filter permeability. Environ. Prot. Eng. 39(2), 77-89.

23. Spychała M., Nieć J., Pawlak M. 2013. Preliminary study on filamentous particle distribution in septic tank effluent and their impact on filter cake development. Environ. Technol. 34(20), 2829-2837.

24. Spychała M., Łucyk P. (2015, in Press). Effect of thickness of textile filter on organic compounds and nutrients removal efficiency at changeable wastewater surface level. Sci. Nat. Technol. 